\title{
Cross-scale Integrated Biomaging by 3D Light-, X-ray and Electron Microscopy - From Organisms, Organs and Tissue to Cells, Organelles and Macromolecular Complexes
}

\author{
Manfred Auer, Ph.D. \\ Cellular and Tissue Imaging Department, Molecular Biophysics and Integrated Bioimaging \\ Division, Lawrence Berkeley National Laboratory, Berkeley, CA 94720
}

We need to understand (loss of) biological function not only at the level of macromolecular complexes and their supramolecular organization but also need to see the impact at the level of organelles, cells, and tissues (possibly even organs and the entire organism). The entire range of scales spans at least six orders of magnitude, ranging from sub-nanometer to millimeters, and thus cannot be imaged by a single imaging modality alone. With each modality providing different types of information about the system at different scale and resolution, it can be challenging to view and comprehend high resolution information in its wider range context. When attempting to map localization signals from fluorescence (optical) microscopy unto ultrastructure determined by electron microscopy, it can be challenging to find the exact same regions and overlay the information, particularly in complex tissues. X-ray microscopy tomography has proven to be an ideal technique to link optical and electron microscopy. In itself the technique provides valuable information about the organ of interest, its tissue organization as well as allows one to visualize and identify individual cells. I will show how we were able to identify individual cells inside a complex vertebrate animal (zebrafish larvae), starting with a fixed, stained and resin-embedded whole-mount larvae, that was imaged by Xray tomography at different scales and resolution. We focused on so-called hair cells residing in the sensory epithelia of the inner ear and underlying our senses of hearing and balance, where they transduce mechanical signals (pressure waves/sound) to electrical signals, courtesy of stereocilia-based hair bundle. We have resolved the 3D organization of individual intact cryopreserved stereocilia, using cryo-electron tomography of vitrified whole mount stereocilia at the macromolecular complex level and have begun to 3D reconstruct 3D hair cells using focused ion beam scanning electron microscopy (FIBSEM). As a second example I will show our results on the structure determination of Arabidopsis thaliana plant cell walls via cryoelectron tomography of vitreous section, and the resulting surprising mechanical properties of 3D organization. Last, I will discuss multimodal imaging results of a cancer progression model and the role of the cytoskeleton of a direct mechanical readout of its microenvironment, and end with a vision on "integrated bioimaging" with an emphasis on geo-/volumetric model generation and extensive quantitative analysis, which -if adopted- we submit will have a major impact on biological imaging. 